\title{
A New Thermodynamic Approach to the Microindentation Process on Polymeric Crystals
}

\author{
Matsuo Hirami, ${ }^{\dagger}$ Francisco Jose Baltá Calleja, ${ }^{*}{ }^{\dagger}$ and Araceli Flores* \\ Research and Development Center, Unitika Ltd., 23 Kozakura, Uji, Kyoto 611-0021, Japan \\ * Instituto de Estructura de la Materia, CSIC, Serrano 119, 28006 Madrid, Spain
}

(Received December 3, 1998)

\begin{abstract}
A new thermodynamic derivation of the microhardness dependence on crystal thickness is developed. The approach makes use of a modified Clausius-Clapeyron equation to incorporate the effect of the finite size of polymer crystals. The derived equation describes the hardness depression due to finite thickness of the lamellae on the assumption that plastic deformation involves a partial melting of the polymer crystals. The present model approach offers an alternative view to the earlier concept of plastic deformation related to the energy dissipated during mechanical destruction of the crystals.

KEY WORDS Microhardness / Polymer Crystals / Crystal Hardness / Crystal Thickness / Crystal Destruction / Partial Melting / Clausius-Clapeyron Equation /
\end{abstract}

In recent years microhardness has been shown to be a powerful technique to detect morphological and microstructural changes in polymers. ${ }^{1,2}$ This technique has been successfully applied to the study of the crystallization process in amorphous polymers. ${ }^{3}$ Baltá Calleja and Kilian ${ }^{4}$ developed an approach based on a heterogeneous deformation model involving the heat dissipated by the plastically deformed polymer crystals to calculate the dependence of hardness on average thickness $l_{\mathrm{c}}$ of the crystalline lamellae. The crystal hardness $H_{\mathrm{c}}$ can then be described by:

$$
H_{\mathrm{c}}=\frac{H_{\mathrm{c}}^{\infty}}{1+\left(b / l_{\mathrm{c}}\right)}
$$

where $H_{c}^{\infty}$ is the hardness of an infinitely thick crystal and $b$ is a parameter given by:

$$
b=2 \sigma_{\mathrm{e}} / \Delta h
$$

$\sigma_{\mathrm{e}}$ being the surface free energy and $\Delta h$ the heat dissipated as a consequence of the plastic deformation of the crystalline blocks. It has already been indicated the existing similarity between eq 1 and the well known Thomson-Gibbs equation. ${ }^{5}$ The latter describes the melting point depression, $T_{\mathrm{m}}^{0}-T_{\mathrm{m}}$, for a polymeric crystal of finite lamellar thickness $l_{\mathrm{c}}$ with respect to the equilibrium melting point $T_{\mathrm{m}}^{0}$ :

$$
T_{\mathrm{m}}=T_{\mathrm{m}}^{0}\left(1-\frac{2 \sigma_{\mathrm{e}}}{\Delta h_{\mathrm{f}}^{0} l_{\mathrm{c}}}\right)
$$

where $\Delta h_{\mathrm{f}}^{0}$ is the equilibrium melting enthalpy.

The aim of this note is to present an alternative model for the derivation of eq 1 based, as well, on thermodynamic considerations. Let us assume that the indentation process induces a partial melting of the polymer crystals during the indentation process. Thus,

$\Delta h$ in eq 2 should be regarded, in this case, as the heat dissipated through the partial melting of the polymer crystals near the sample surface. Let us make use of the Clausius-Clapeyron equation, which is applicable to crystalline polymer systems. This equation describes the

\footnotetext{
$\dagger$ To whom all correspondence should be addressed
}

increase in melting point as a consequence of an increasing pressure $p$ :

$$
T_{\mathrm{m}}^{\mathrm{o}}\left(\frac{\mathrm{d} p}{\mathrm{~d} T_{\mathrm{m}}^{0}}\right)=\frac{\Delta h_{\mathrm{f}}^{0}}{\Delta V_{\mathrm{m}}^{0}}
$$

where $\Delta V_{\mathrm{m}}^{0}$ is the specific volume change upon melting for an infinitely thick crystal. Equation 4 may be applied to finite size polymer crystals taking into account the following considerations:

i) $\Delta h_{\mathrm{f}}^{0}=\Delta h_{\mathrm{f}}-2 \sigma_{\mathrm{e}}^{\mathrm{H}} / l_{\mathrm{c}}$, where $\Delta h_{\mathrm{f}}$ is the enthalpy of fusion of a crystal of thickness $l_{\mathrm{c}}$ and fold surface enthalpy $\sigma_{\mathrm{e}}^{\mathrm{H}}$. The surface free energy is the sum of an enthalpy and an entropy term, i.e., $\sigma_{\mathrm{e}}=\sigma_{\mathrm{e}}^{\mathrm{H}}-T \sigma_{\mathrm{e}}^{\mathrm{S}}$. Since, usually, $\sigma_{\mathrm{e}}^{\mathrm{H}} \gg T \sigma_{\mathrm{e}}^{\mathrm{s}}$, we may use the approximation $\sigma_{\mathrm{e}}^{\mathrm{H}} \cong \sigma_{\mathrm{e}}$.

ii) $\Delta V_{\mathrm{m}}^{\mathrm{o}} \cong \Delta V_{\mathrm{m}}$ (where $\Delta V_{\mathrm{m}}$ is the specific volume change upon melting of a crystal with finite thickness).

iii) From eq $3, T_{\mathrm{m}}^{0} / \mathrm{d} T_{\mathrm{m}}^{0}=T_{\mathrm{m}} / \mathrm{d} T_{\mathrm{m}}$.

By combination of i), ii), and iii), one can obtain:

$$
T_{\mathrm{m}}\left(\frac{\mathrm{d} p}{\mathrm{~d} T_{\mathrm{m}}}\right)=\left(\frac{\Delta h_{\mathrm{f}}}{\Delta V_{\mathrm{m}}}\right)\left(1-\frac{2 \sigma_{\mathrm{e}}}{\Delta h_{\mathrm{f}} l_{\mathrm{c}}}\right)
$$

Let us now apply eq 5 to an indentation experiment. Integration of eq 5 from the reference state 1 (prior to indentation) to the state 2 (where the indenter penetrates the sample) yields:

$$
p_{2}-p_{1}=K_{\mathrm{f}}\left(1-\frac{2 \sigma_{\mathrm{e}}}{\Delta h_{\mathrm{f}} l_{\mathrm{c}}}\right)
$$

where $p_{1}=0, p_{2}$ corresponds to the pressure just after penetration, and

$$
K_{\mathrm{f}}=\left(\frac{\Delta h_{\mathrm{f}}}{\Delta V_{\mathrm{m}}}\right) \ln \left(\frac{T_{\mathrm{m}(2)}}{T_{\mathrm{m}(1)}}\right)
$$

Since we are concerned here with the changes induced on the fraction of crystalline material upon indentation, $\left(p_{2}-p_{1}\right)=p_{2}$ is directly related to the crystal hardness $H_{\mathrm{c}}$. It should be recalled that the hardness is a measure of the mean contact pressure under the indenter. ${ }^{6}$ Hence, $H_{\mathrm{c}}=p_{2}$. The enthalpy of fusion $\Delta h_{\mathrm{f}}$ in eq 6 should be seen as the enthalpy change $\Delta h$ accompanying the partial melting of the crystals upon indentation. Furthermore, 
the increase in temperature $\left(T_{\mathrm{m}(2)}-T_{\mathrm{m}(1)}\right)$ is a consequence of the heat dissipated during this process. Hence, one could rewrite eq 6 as:

$$
H_{\mathrm{c}}=K\left(1-\frac{2 \sigma_{\mathrm{e}}}{\Delta h l_{\mathrm{c}}}\right) \approx \frac{K}{1+\left(2 \sigma_{\mathrm{e}} / \Delta h l_{\mathrm{c}}\right)}
$$

where

$$
K=\left(\frac{\Delta h}{\Delta V_{\mathrm{m}}}\right) \ln \left(\frac{T_{\mathrm{m}(2)}}{T_{\mathrm{m}(1)}}\right)
$$

expression which is identical to eq 1 if $K$ is identified with $H_{\mathrm{c}}^{\infty}$. It should be noted that according to both models (mechanical deformation and partial melting models), $\Delta h$ should be smaller than $\Delta h_{\mathrm{f}}$. Indeed, we have shown that for polyethylene and poly(ethylene oxide) crystals $\Delta h \ll \Delta h_{\mathrm{f}} .{ }^{5,7}$

In conclusion, the thermodynamic approach used, based on the assumption of partial melting of crystals upon indentation, leads to the same dependence of hardness on crystalline lamellar thickness (eq 1) as previously derived. Whether partial melting or the mechanical breaking of crystals is involved during indentation is still an open question. Further clarification to the problem will be attempted experimentally by means of real time microdiffraction studies during indentation. ${ }^{8}$

Acknowledgments. Grateful acknowledgment is due to the international joint research grant NEDO and to the DGICYT, Spain (Grant PB94-0049) for the support of this investigation. One of us (M.H.) is indebted to Prof. L. Mandelkern for his valuable comments concerning the derivation of eq 5 .

\section{REFERENCES}

1. F. J. Baltá Calleja, Trends Polym. Sci., 2, 419 (1994).

2. Y. Deslandes, E. Alva Rosa, F. Brisse, and T. Meneghini, $J$. Mater. Sci., 26, 2769 (1991).

3. F. J. Baltá Calleja, C. Santa Cruz, and T. Asano, J. Polym. Sci., Polym. Phys. Ed., 31, 557 (1993).

4. F. J. Baltá Calleja and H. G. Kilian, Colloid Polym. Sci., 266, 29 (1988).

5. F. J. Baltá Calleja, C. Santa Cruz, R. K. Bayer, and H. G. Kilian, Colloid Polym. Sci., 268, 440 (1990).

6. D. Tabor, "Gases, Liquids and Solids," 2nd ed, Cambridge University Press, Cambridge, 1979.

7. F. J. Baltá Calleja and C. Santa Cruz, Acta Polym., 47, 303 (1996).

8. C. Riekel, F. J. Baltá Calleja, and A. Flores, in preparation. 